\title{
Investigation of QoS Multicast Routing Based on Intelligent Multiple Constrained
}

\author{
Firas Mahdi Muhsin Al-Salbi \\ Electrical \& Electronics Dept., Engineering College, Kerballa University, Kerballa, Iraq
}

Received: June 1, 2011

Accepted: June 20, 2011

doi:10.5539/cis.v4n4p64

\begin{abstract}
With the rapid development of Internet, mobile networks and high-performance networking technology, quality-of-service (QoS) of multicast routing has become a very important research issue in the areas of networks and distributed systems. This paper introduces quality of services multicast routing by using intelligent Algorithm. Its main objective is to construct a multicast tree that optimizes a multi objective function with respect to performance-related constraints (Short path, delay, packet loss). The proposed algorithm can be divided in two steps. First step, multicast tree selection algorithm based on shortest path. Second step, to find routes with two or more QoS parameters is a hard problem. Therefore, it will divide in two parts according to the customer service. Part one; optimize QoS Based on delay minimization and packet loss. Part two; optimize QoS Based on cost minimization and packet loss. The simulation results show that the proposed algorithm is effective approach to multicast routing decision with multiple QoS constrains for mobile ad-hoc networks.
\end{abstract}

Keywords: QoS, Multicast Routing, Shortest path, Delay

\section{Introduction}

Multicast services have been used by a variety of continuous many applications. With the development of network technology with high performance such as Multicasting Network, the study of design theory and methodology based on QoS multicast routing protocol has been an important subject in network study. Therefore, the multicasting plays a significant role in the networks. QoS multicast routing relies on state parameters specifying resource availability at network nodes or links, and uses them to find paths with enough free resources. The problem of QoS multicast routing with multiple constrains is an NP complete problem (Chen Niansheng, et al.,2006; Chen Niansheng, et al. ,2006), some heuristics is being used to solve this problem.

Recently, many researches focus more in determining QoS based multicast routes clearly demonstrate the power of genetic algorithms to get a near-optimal solution satisfying the QoS requirements in computationally feasible time (LI La-Yuan,LI Chun-Lin, 2004; Jain, S., Sharma, J.,2008; Yang H S, et al.,2003; Glover F, et al.,1995). Traditionally, a network has been modeled as a directed graph and the QoS parameters are specified by a fixed metric(s) assigned to the edges (Molnar, M.,2009; Ali, N.A et al.,2005; Charikar M., et al. ,2004; Fu-sheng Dai and Ai-jun Liu,2009). When a more complex problem must be faced, e.g., the Steiner tree problem (STP), other types of algorithms, which can solve these multicast problems, must be considered. Furthermore, for multimedia routing QoS parameters such as communication delay, cost, bandwidth and so on should be considered (Kawano K., et al.,2001). The classical approach is based on polynomial algorithms such as Dijkstra or Prim, which resolve problems when only a QoS metric is considered (Wang B. and J. C. Hou,2000; Winter P. , 1987). For interactive real-time traffic, the delay-constrained least-cost path has particular importance (Lin H, et al., 2007). It is the cheapest path whose end-to-end delay is bounded by the delay requirement of a time-sensitive data flow. (Salama H.F., et al. 1997, Xue G.,2000) used the Lagrange relaxation method to approximate the delay-constrained least-cost routing problem. However, there is no theoretical bound on how large the cost of the found path. Therefore, some researchers have started using evolutionary algorithms to find near-optimal solutions for different networking problems, like QoS-Routing (Juttner A., et al. 2001; Xiang F, et al.2003; Li Dawei, Wang Mengguang ,1997). For example, the multicast backbone of the Internet has been used to transport real time audio/video for news, entertainment, video conferencing, and distance learning (Charikar M, et al. 2000; Roch A G and Orda A, 1999; Liu Ying, Wu Jianping 2003). Korkmaz and Krunz used a nonlinear target function to approximate the multi-constrained least-cost path problem (Wang Bin and Hou JC 2000). It was proved that the path that minimizes the target function satisfies one constraint and the other constraints multiplied by, where is a predefined constant and is the number of constraints. 
This paper introduces two techniques (delay minimization and Cost minimization) based on customer service which allows faster intelligent algorithms to be designed. Also, the proposed algorithm focus on reducing the overhead of computing constrained shortest paths is practically important for the successful design of a high-throughput QoS router with respect to performance-related constraints (delay, cost, packet loss). Simulation results show that the algorithm is an effective approach to the problem of multicast routing decision with multiple QoS constraints.

The structure of this paper is as follows. Sections two introduce related work. Section three discusses QoS network model and routing problem. Section four describes the proposed QoS algorithm. In Section five, discuss Simulation result. Finally, some conclusions are given in Section six.

\section{Related Work}

Various classes of algorithms with different development techniques have been proposed. Some of them were developed with heuristic techniques, because these problems have a higher computational complexity. The provision of Quality-of-Service (QoS) guarantees is of utmost importance for the development of the multicast services (Jain, S.; Sharma, J. 2008; Yang H S, et al. 2003; Glover F, et al. 1995). Multicast routing has continued to be a very important research issue in the areas of networks and distributed systems. It attracts the interests of many people. QoS multicast routing relies on state parameters specifying resource availability at network nodes or links, and uses them to find paths with enough free resources. Multicast problems, with a lower complexity, consider simple routing with only one quality-of service (QoS) metric; thus, they can be solved with algorithms such as Prim, Dijkstra, or Kruskal (Chen Niansheng, et al. 2006; Chen Niansheng, et al. 2006). When an STP (LI La-Yuan,LI Chun-Lin 2004) is considered, an NP-complete problem must be faced. In this case, to solve the multicast routing problem, alternative approaches could be used. These methods permit good solutions close to the optimum solution that needs to be found. The classical approach is based on polynomial algorithms such as Dijkstra or Prim, which resolve problems when only a QoS metric is considered (Molnar, M.,2009; Ali, N.A et al.2005). When a more complex problem must be faced, e.g., the Steiner tree problem (STP), other types of algorithms, which can solve these multicast problems, must be considered.

At present, Link expiration time model predicts the connect time between two nodes according to the node's information (such as location, velocity, direction etc). Multicast routing protocols and algorithms are designed to construct and maintain a tree structure; moreover, they provide for complete support to member joining/leaving and maintaining updated information. Moreover, due to QoS issues, many multicast protocols have been considered in the literature (Chen Niansheng, et al. 2006; Chen Niansheng, et al. 2006). QoS IP multicast involves the construction and maintenance of trees that reflect a desired service quality. The QoS routing problem formulated in this manner is equivalent to Minimum Steiner Tree problem, which is NP-complete (Yang H S, et al. 2003). A large number of approximate algorithms have been suggested by researchers for the above problem (Glover F, et al. 1995). In some cases, exact algorithms with exponential time complexity have been proposed (Lin H, et al. 2007). In the existing work on QoS multicasting both the allocated resources and the metrics are assumed to be static and cannot be changed during the process of path hunting. In addition, the results of the above mentioned approaches do not incorporate the effect of multiple routers, connected in tandem, on the QoS parameters of a path, rather the QoS metrics are simply added. Also, these results do not include the possibility of tuning the allocated resources during the path search in order to establish a multicast tree that satisfies all the QoS requirements collectively. For example, suppose that for a particular connection the jitter requirement is very tight while the data droppage requirement has some flexibility. In this case, it may be essential to reduce the queue size to achieve reduced jitter caused by the queuing delay, even though abundant memory may be available. In other words, assigning the metrics prior to the path searching algorithm may preclude finding a tree that satisfies the QoS requirements, even though when one such tree may exist. In summary, existing results do not provide a single framework that allows routers and switches to utilize the combined knowledge of hardware/software implementation and the available resources to take intelligent routing decisions.

\section{QOS network Model and Routing Problem}

\section{A) QOS MULTICAST MODEL}

Fig.1 show Connection the source has the entire network state information (cost, delay, etc.. of each edge) With some QoS requirements. 


\section{1) Network Model}

The network in fig.1 is modeled as a set of nodes that are usually represented as a weighted digraph $G=(V, E)$, where $\mathrm{V}$ denotes the set of nodes and $\mathrm{E}$ denotes the set of communication links connecting the nodes. $|\mathrm{V}|$ and $|\mathrm{E}|$ denote the number of nodes and links in the network, respectively. Without loss of generality, only digraphs are considered in which there exists at most one link between a pair of ordered nodes (Ali, N.A et al. 2005). Associated with each link are parameters that describe the current status of the link.

Let $\mathrm{s} \in \mathrm{V}$ be source node of a multicast tree, and $\mathrm{M} \subseteq\{\mathrm{V}-\{\mathrm{s}\}\}$ be a set of end nodes of the multicast tree.

Let $\mathrm{R}$ be the positive weight and $\mathrm{R}+$ be the nonnegative weight. For any Link $\mathrm{e} \in \mathrm{E}$, we can define the some QoS metrics: delay function delay (e): $\mathrm{E} \rightarrow \mathrm{R}$, cost function cost (e):

$\mathrm{E} \rightarrow \mathrm{R}$, bandwidth function bandwidth (e): $\mathrm{E} \rightarrow \mathrm{R}$, and delay jitter function delay-jitter (e): $\mathrm{E} \rightarrow \mathrm{R}+$.

For any node $n \in V$, one can also define some metrics:

delay function delay $(\mathrm{n}): \mathrm{V} \rightarrow \mathrm{R}$, cost function cost $(\mathrm{n}): \mathrm{V} \rightarrow \mathrm{R}$, delay jitter function delay-jitter $(\mathrm{n}): \mathrm{V} \rightarrow \mathrm{R}+$ and packet loss function packet-loss $(\mathrm{n}): \mathrm{V} \rightarrow \mathrm{R}+$.

We also use $\mathrm{T}(\mathrm{s}, \mathrm{M})$ to denote a multicast tree, which has the following relations:

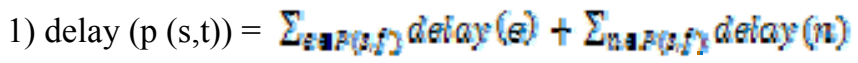

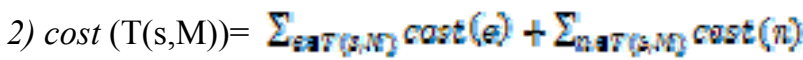

3) bandwidth $(\mathrm{p}(\mathrm{s}, \mathrm{t}))=\min \{$ bandwidth $(\mathrm{e}), \mathrm{e} \in \mathrm{P}(\mathrm{s}, \mathrm{t})\}$.

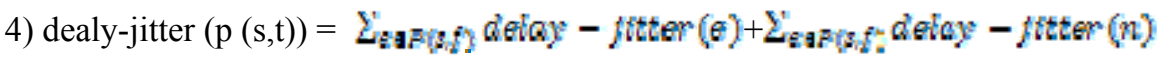

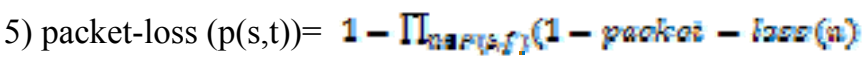

where $\mathrm{p}(\mathrm{s}, \mathrm{t})$ denotes the path from source $\mathrm{s}$ to end node $\mathrm{t}$ of $\mathrm{T}(\mathrm{s}, \mathrm{M})$.

2) Stationary and transient Links

Transient Links: links between nodes that move fast, exists only for a short time.

Stationary: links between nodes that move slowly, assuming continuous connection.

3) QOS State Matrix

A node i has a precise information about it's neighbors:

$$
\begin{aligned}
& \text { a. delay }(i, j) \text {. } \\
& \text { b. bandwidth }(i, j) . \\
& \text { c. cost }(i, j) . \\
& \quad(\text { transient link cost much more) }
\end{aligned}
$$

Path $\mathrm{P}=\mathrm{i}->\mathrm{j}->\ldots$.. $>\mathrm{k}->1$.

Delay $(\mathrm{P})=\operatorname{delay}(\mathrm{i}, \mathrm{j})+\ldots+\operatorname{delay}(\mathrm{k}, \mathrm{l})$.

Bandwidth $(\mathrm{P})=$ $\min \{$ bandwidth(i,j),..,bandwidth $(\mathrm{k}, \mathrm{l})\}$.

$\operatorname{Cost}(\mathrm{P})=\operatorname{cost}(\mathrm{i}, \mathrm{j})+\ldots+\operatorname{cost}(\mathrm{k}, \mathrm{l})$.

B) The Routing Problem

QoS-based multicast routing problem mainly deals with some elements: Network $G=(V, E)$, multicast source $\mathrm{s} \in \mathrm{V}$, the set of end nodes $\mathrm{M} \subseteq\{\mathrm{V}-\{\mathrm{s}\}\}$, delay $(\cdot) \in \mathrm{R}$, delay-jitter $(\cdot) \in \mathrm{R}+$, cost $(\cdot) \in \mathrm{R}$, bandwidth $(\cdot) \in \mathrm{R}$, and packet-loss $(\cdot) \in \mathrm{R}+$.

This routing problem is to find the $\mathrm{T}(\mathrm{s}, \mathrm{M})$ which satisfies some QoS constraints:

1) Delay constraint: delay $(\mathrm{p}(\mathrm{s}, \mathrm{t})) \leq \mathrm{D}$

2) Bandwidth constraint: bandwidth $(p(s, t)) \geq B$

3) Delay jitter constraint: delay-jitter $(p(s, t)) \leq J$

4) Packet loss constraint: packet-loss $(\mathrm{p}(\mathrm{s}, \mathrm{t})) \leq \mathrm{L}$

Meanwhile, the cost (T (s, M) should be minimum. Where D is delay constraint, B is bandwidth constraint, $\mathrm{J}$ is delay jitter constraint and $\mathrm{L}$ is packet loss constraint. In the above QoS constraints, the bandwidth is concave metric, the delay and delay jitter are additive metrics, and the packet loss is multiplicative metric. 
The routing problem can be summarized as follows.

1) Delay Constrained Routing Problem

To find a feasible path $\mathrm{P}$ from $\mathrm{S}$ to $t$ such that $\operatorname{delay}(\mathrm{P}) \leq \mathrm{D}$, when there are multiple such paths, select the one with least delay.

2) Bandwidth Constrained Routing

To find a feasible path $\mathrm{P}$ from $\mathrm{S}$ to $t$ such that bandwidth $(\mathrm{P}) \geq \mathrm{B}$, when there are multiple such paths, select the one with least cost.

Finally, to collectively utilize the state information at the intermediate nodes to guide the routing messages along the best paths to the destination, so that the probability of finding feasible low-cost path is maximized.

- A source node issues a number of tickets based on the available state information.

- Each routing message contains at least one ticket.

- A probe can be split by intermediate node if it contains more than one ticket.

- The total number of messages is bounded by the number of tickets issued by the source node.

- An Intermediate node decides whether an incoming probe should be split and to which of its neighbors to forward the message.

\section{The proposed method}

The algorithm is given in a general form, and relies on the sub-algorithms. This algorithm realizes the routing with two parts. First, shortest path in minimum cost for the constrained delay time. Second, shortest path in minimum delay time for the constrained cost. The outline of this algorithm is explained in following.

The main algorithm starting from a tree with only s

- Higher-layer receiver i first

- Select the most appropriate path P from T

- A setup message is sent to $i$ along P, carrying the data structure RECEIVER and D(delay)

- RECEIVER is updated by intermediate nodes, if better path is available

- Next off-tree receiver $\mathrm{j}$ is selected by $\mathrm{i}$

- A fork message is sent from $\mathrm{i}$

- A finish message is sent to $s$ if no off-tree node

Sub algorithm Path Selection from T

- Calculate the minimum shortest path $\mathrm{r}_{\mathrm{i}}: \quad \mathrm{r}_{\mathrm{i}}>=\left(\operatorname{sigma}^{\mathrm{k}}+|\mathrm{p}|^{*} \mathrm{c}\right) /\left(\mathrm{D}_{\mathrm{i}}-\operatorname{sum}\left(\mathrm{d}_{1}\right)\right)$

- Select the least-hop path with $\mathrm{T}(\mathrm{i}, \mathrm{h}) \cdot \mathrm{bw}>=\max \left(\mathrm{r}_{\mathrm{i}}, \mathrm{R}^{\mathrm{k}}\right)$

- No loop

- Reserved shortest path: $\max \left(\mathrm{r}_{\mathrm{i}}, \mathrm{R}^{\mathrm{k}}\right)$

- if no path exists, or $\mathrm{r}_{\mathrm{i}}>\mathrm{R}_{\mathrm{i}}^{\mathrm{r}}$, degrading layer

(Best path?)

First Part: Sub Algorithm Minimum Cost

- OnTreeNode: initialized to $\mathrm{s}$

- path: P, with sufficient shortest path

- $\mathrm{R}^{\mathrm{r}}$ : the minimum rate for delay

- cost: $|\mathrm{P}|^{*} \mathrm{R}^{\mathrm{r}}$, the total cost

Second Part: Sub Algorithm Minimum delay

- OnTreeNode: initialized to $\mathrm{s}$

- path: $\mathrm{P}$, with sufficient shortest path

- $t$ : the minimum time for cost

- delay: $|\mathrm{P}|^{*} \mathrm{t}$, the total cost

Path Update

Intermediate nodes update: 
- Delay requirement (D:cumulative delay) $\mathrm{D}_{\mathrm{i}}>=\mathrm{D}+\left(\operatorname{sigma}^{\mathrm{k}}+|\mathrm{p}| * \mathrm{c}\right) / \mathrm{r}_{\mathrm{i}}+\operatorname{sum}\left(\mathrm{d}_{1}\right)$

- Select the minimum-hop path P from T(first entry T(i,h))

- Lowest cost: $|\mathrm{P}|^{*} \mathrm{R}^{\mathrm{r}}$

Prior to executing the main loop, the sub-algorithm Pre-Process performs some preliminary processing such as deleting edges with insufficient QOS.

\section{Simulation Result}

The simulations run with a constant number of $25 \%$ edge nodes (as opposed to the $20 \%$ we usually used). The number of candidate destination nodes varied from $1 \%$ up to the whole set of edge nodes $(25 \%)$. The candidate set of source nodes was always the whole set of edge nodes. Only the links from those destination nodes were assigned a bandwidth capacity of $1000 \mathrm{Mb}$.

The success ratio for different node delays and tree sizes for simulations with delay QoS requirements are presented in Figure 2. As the same simulation parameters are used for the flat as well as hierarchical routings, the success ratio has similar behavior in both cases. The path delay of Flooding messages increases with the node delay. Therefore, for a given tree size, the number of messages rejected by the QoS forwarding condition increases and, hence, the success ratio decreases with an increase in the node delay. Since the average path length of Flooding messages is higher for fewer on-tree routers, this effect is more prominent for 30-node trees than in the other case (Figure 2).

The success ratio for different delay requirements is presented in Figure 3. As in Figure 2, the performance of hierarchical and flat routing protocols is similar. For a given tree size, the number of Flooding messages rejected by the QoS forwarding conditions decreases and, hence, success ratio increases with an increase in the delay requirement. The average path length of Flooding messages decrease with an increase in tree size. Therefore, for a given node delay, the path delay and the number of messages rejected by the QoS forwarding conditions decreases and, hence, the success ratio increases with an increase in tree size (Figure 3).

The characteristic of computation time versus delay constraint for 30 nodes is shown in Fig. 4. From the figure, it is clearly that the computation time of proposed algorithm is lower than computation time of the conventional algorithm. The computation time for the minimum path is decreased by selecting first the largest sub-tree. This is why the computation time of proposed algorithm is smaller than the conventional algorithm. The characteristic of search success ratio versus delay constraint for 60 nodes is shown in Fig. 6. Also, in this case, the proposed protocol shows better performance than conventional algorithm the same as in Fig. 4. However, the difference between characteristics of the proposed algorithm and the conventional algorithm is larger than the case with 30 nodes. The characteristic of computation time versus delay constraint for 60 nodes is shown in Fig. 5. In this case, the proposed protocol has better behavior than the conventional algorithm like the characteristics of Fig. 5 . But, the difference between characteristics of the proposed algorithm and the conventional algorithm is larger than the case with 30 nodes. From all results, it concluded that the proposed algorithm has better characteristics than the conventional algorithm.

\section{Conclusion}

QoS multicast routing is the foreland research project in networks and information technology. While interests of many people have got better results in single, this paper study QoS multicast routing model and optimization algorithm based on delay and cost constraints and gives the heuristic genetic algorithm of minimum-costs QoS multicast tree and cost-delay constraint. The feature that makes it distinct from previous formulations is that the QoS parameters of the links are taken to be functions, rather than fixed value metrics. The new formulation has the advantage of using the knowledge of the hardware and software of the nodes to intelligently satisfy the multiple conflicting QoS requirements of the end users. The experiment represents that its convergent speed is fast and reliability. Especially in large network, the algorithm can greatly decrease routing computation time, satisfying the topology structure of real-time communication environment, high dynamic and the requirement of network structure of QoS multicast routing.

\section{References}

Chen Niansheng, Li Layuan,Dong Wushi (2006). A Multicast Routing Algorithm of Multiple QoS Based on Widest-Bandwidth. Journal of Systems Engineering and Electronics, 17(3):642-647.

Chen Niansheng, Li Layuan,Chen Chuanhui. (2006). QoS Multicast Routing Algorithm based on Layered Structure. In Proceedings of the 2006 International Symposium on Distributed Computing and Applications to Business Engineering and Science, pp.1135-1139. 
LI La-Yuan,LI Chun-Lin. (2004). A Multicast Routing Protocol with Multiple QoS Constraints. Journal of Software, 15(2):286 -291.

Jain, S.; Sharma, J. (2008). Delay bound multicast routing algorithm using evolutionary programming Networks, 2008. 16th IEEE International Conference on ICON ,1 - 4 .

Yang H S, Maier M, Reisslein M and Carlyle W M. (2003). A Genetic Algorithm based Methodology for Optimizing Multi- Service Convergence in a Metro WDM Network. IEEE/OSA Journal of Lightwave Technology, 21(5):1114-1133.

Glover F, Kelly J, L aguna M. (1995). Genetic algorithms and tabu search: Hybrids for optimizations. Computer Ops Res, 22(1):111-134.

Molnar, M. (2009). On the Optimal Tree-Based Explicit Multicast Routing. Second International Conference on Communication Theory, Reliability, and Quality of Service, 91 - 96.

Ali, N.A.; Mouftah, H.; Gazor, S. (2005). A distributed bandwidth-guaranteed routing algorithm for point-to-multipoint VPLS virtual connections. IEEE International Conference on Communications, 3: 1561 1565 .

Charikar M., J. Naor and B. Schieber. (2004). Resource optimization in QoS multicast routing of real-time multimedia. IEEE/ACM Transactions onNetworking, 12(2):340-348.

Fu-sheng Dai; Ai-jun Liu. (2009). A Multi-Constrained Quality of Service Routing Algorithm Based on Vector Converting. 25th International Conference on Wireless Communications, Networking and Mobile Computing , 1 -4 .

Kawano K., T. Masuda, K. Kinoshita and K. Murakami. (2001). An Efficient Method to Search for the Location of Network Services with Multiple QoSs Guarantee. Transaction of IEICE, J84-B(3):443-451.

Wang B. and J. C. Hou. (2000). Multicast routing and its QoS extension: Problems, algorithms, and protocols. IEEE Netw., 14(1):22-36.

Winter P. (1987). Steiner problem in networks: A survey. Networks, 17(2):129-167.

Lin H., Z. Yu-Lin, and R. Yong-Hong. (2007). Two multiconstrained multicast QoS routing algorithms, in Proc. 8th ACIS Int. Conf. SNPD, 3:495-500.

Salama H.F., D. S. Reeves, and Y. Viniotis. (1997). A distributed algorithm for delay-constrained unicast routing. In Proc. IEEE INFOCOM, 84-91.

Xue G. (2000). Primal-dual algorithms for computing weight-constrained shortest paths and weight-constrained minimum spanning trees. In Proc. IEEE IPCCC’00, 271-277.

Juttner A., B. Szviatovszki, I. Mecs, and Z. Rajko. (2001). Lagrange relaxation based method for the QoS routing problem. In Proc. IEEE INFOCOM, 859-868.

Xiang F, Junzhou L,Jieyi W,Guanqun G. (2003). An Optimizing on Multiple Constraints QoS Multicast Routing based on Genetic Algorithms. Computer Engineering and Application, 30:1-3.

Li Dawei, Wang Mengguang. (1997). An Improved hybrid Genetic Algorithm Information and Control, 26(6):449-454.

Charikar M, Naor J and Schieber B. (2000). Resource optimization in QoS multicast routing of real-time multimedia. In Proc of IEEE INFOCOM, 1518-1527.

Roch A G and Orda A. (1999). QoS routing in networks with inaccurate information: Theory and algorithms. IEEE/ACM. Trans. On Networking, 3(7):350-363.

Liu Ying, Wu Jianping. (2003). A Delay-Constrained Multicast Routing Algorithm Based on Heuristic Genetic Algorithm. Journal of Computer Research and Development, 40(3):381-386.

Wang Bin and Hou JC. (2000). Multicast routing and its QoS extension: Problems, algorithms, and protocols. IEEE Network, (1,2):22-36.

Avallone, S. (2007). On the setting of QoS link weights to provide per-flow QoS guarantees. 12th IEEE Symposium on Digital Computers and Communications, $443-44$. 


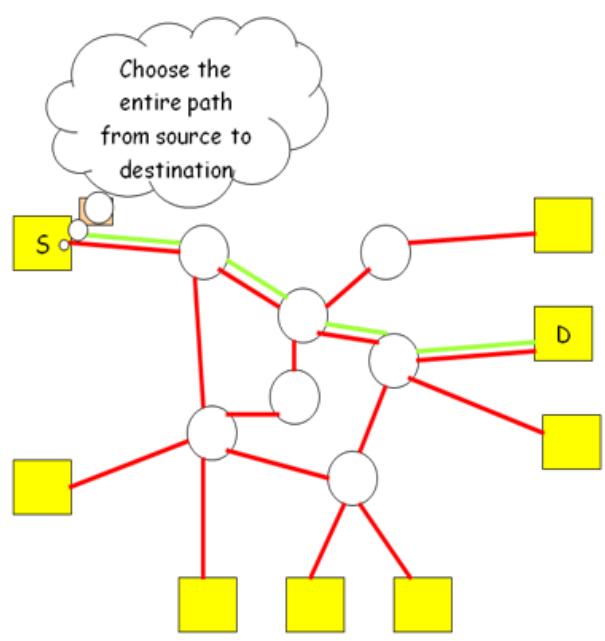

Figure 1. Network connection

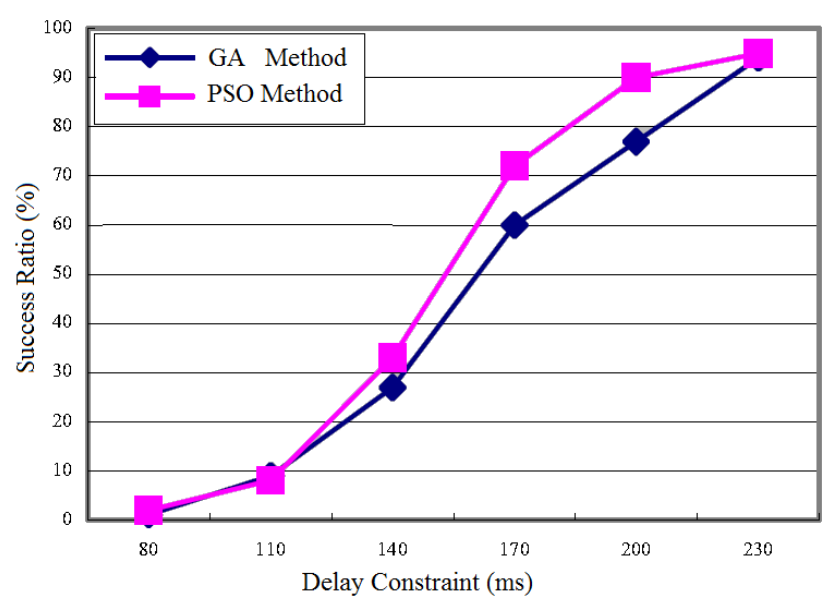

Figure 2. Success ratio for 30 nodes, Cost constraint 2150

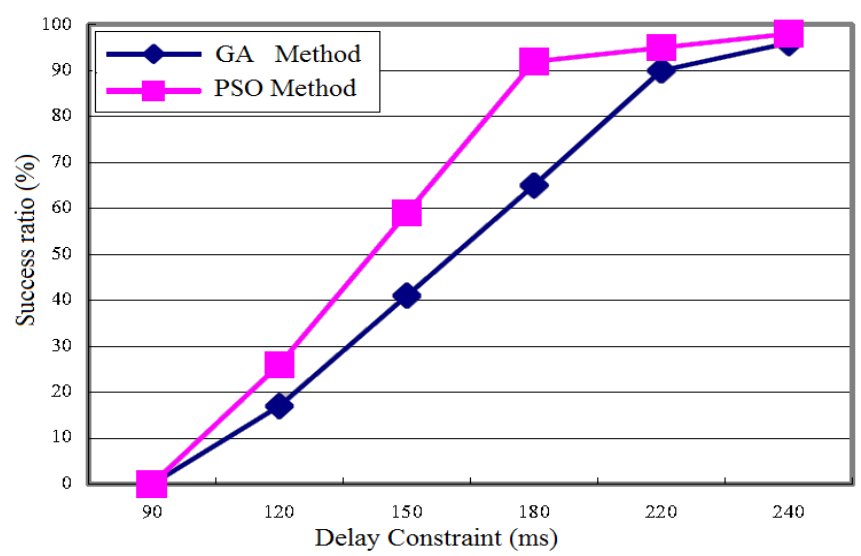

Figure 3. Success ratio for 60 nodes, Cost constraint 2950 


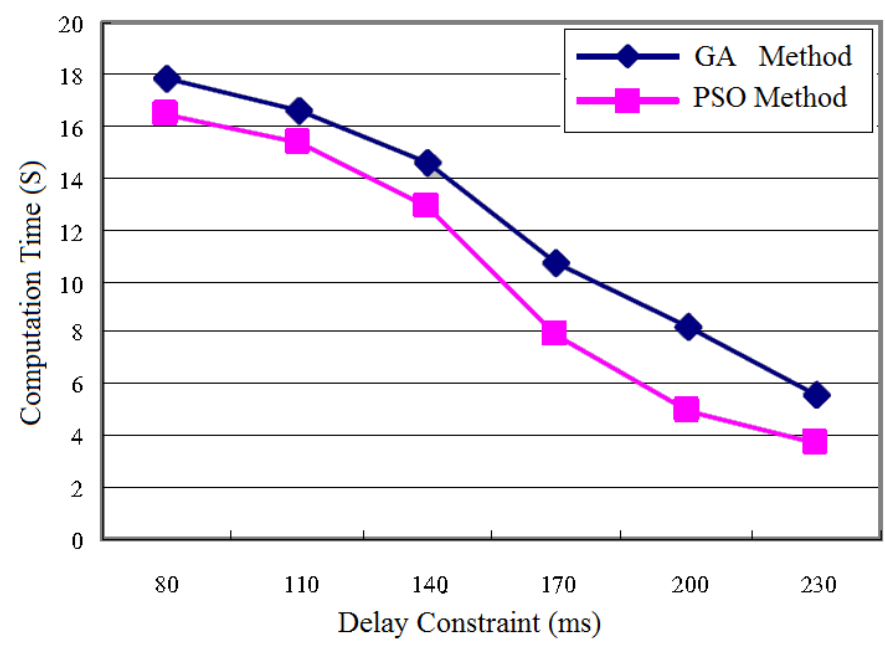

Figure 4. computation time for 30 nodes, Cost constraint 2150

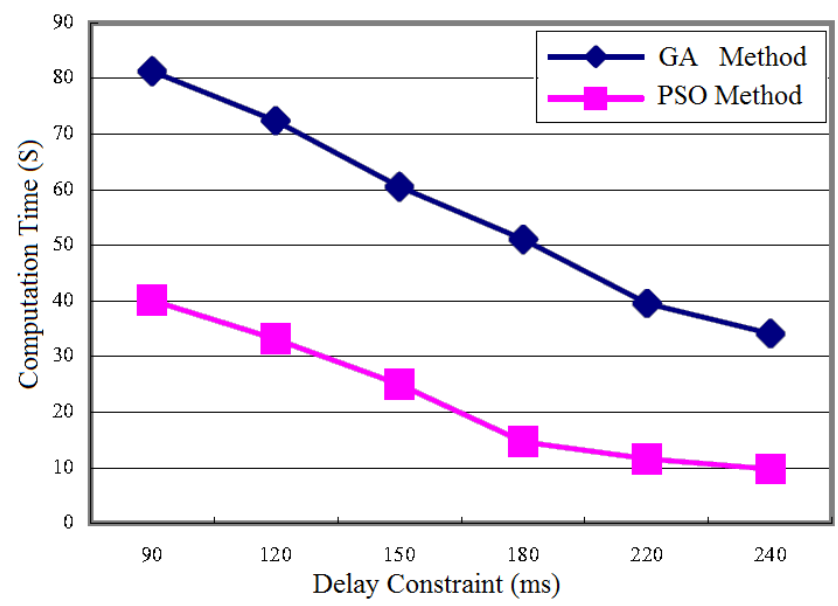

Figure 5. computation time for 60 nodes, Cost constraint 2950 